2.

\title{
Nachtrag zu meinem Artikel „Über multiple Amyloidtumoren des Kehlkopfes und der Lunge".
}

In Band 174, Heft 1 dieses Archivs.

Von

Dr. Gotthold Herxheimer,

Prosektor am Städtischen Krankenhause in Wiesbaden.

Während ich bestrebt war, in meinem in diesem Archiv vor kurzem erschienenen Artikel über Amyloidtumoren die Literatur dieser (mit Ausnahme derer der Conjunctiva) möglichst vollständig zusammenzustellen, labe ich nachträglich noch Kenntnis von einigen wenigen derartigen, früher publizierten Fällen gewonnen, auf welche ich z. T. aufmerksam gemacht wurde. Ich möchte dieselben daher hier noch nachholen.

Zunächst ein Fall, den Krückmann, ein Sehüler Lubarschs, in einem Artikel „Uber Fremdkörper-Tuberkulose und. Fremdkörper-Riesenzellen" in diesem Archiv (Bd. 138, Suppl.) beschrieben. Er faßtihn zwar als ein Endotheliom einer Lymphdrüse mit ausgedehnter hyaliner und amyloider Degeneration auf, jedoch sind die Verhältnisse - vor allem das massenhafte Auftreten von Amyloid, besonders in präformierten Gängen mit Endothelien, aus denen die Fremdkörperriesenzellen hervorgehen, sowie die Übergänge von Hyalin in Amyloid - den sonst beschriebenen Amyloidtumoren so ähnlich, daß wir ihn ruhig diesen zuzählen können, zumal ja auch diese keine scharf begrenzte Gruppe bilden. Interessant in diesem Falle ist noch der Befund amyloider Sehollen und einer mehr diffusen Amyloidreaktion in den Riesenzellen.

Lubarsch selbst hat eine Reihe von in die Gruppe der "Cylindrome" gehörenden Tumoren beschrieben, ${ }^{1}$ ) in welchen er neben "Hyalin" auch "Amyloid" nachweisen konnte. Gehören diese auch nicht ganz in die Gruppe der „Amyloidtumoren“, so möchte ich sie doch deshalb erwähnen, weil Lubarsch auf Grund dieser Fälle besonders auf die nahe - auch färberische - Verwandtschaft nicht nur zwischen Amyloid and Hyalin, sondern auch zwischen Amyloid und Schleim hinwies.

Im Hinblick auf die von mir beschriebenen Amyloidtumoren in der Lunge ist es von Interesse, daß Lubarsch (Lubarsch-Ostertag, Ergebnisse usw., Jahrgang I, Abt. II, S. 216-17) erwähnt, bei einer 75jährigen Fran in der Lunge Knoten gefunden zu haben, „die gröBtenteils aus hyalinen Bindegewebsbalken zusammengesetzt waren, teilweise aber auch amyloide Gewebszüge enthielten".

1) Ergebnisse der Allgem. Path. usw., Jahrg. I, S. 218. - Verhandl. d. Naturf.-Vers. 1896 (Lübeck), Teil 2, Hälfte 2, S. 5. - Ergebnisse d. Allgem. Path. usw., Jahrg. II, S. 597. 
Am interessantesten nun gerade im Hinblick auf den von mir beschriebenen Fall erscheint mir eine Tübinger Dissertation von Burk aus dem Jahre 1901, welche ich in dem Berichte Baumgartens über in seinem Institut ausgearbeitete Dissertationen (Arbeiten usw. aus dem. Pathologisch-anatomischen Institut zu Tübingen, herausgegeben von B a umgarten, 1903, Bd. IV, 2) erwähnt finde. Es handelte sich um einen primären Amyloidtumor der Thyreoidea mit Metastasen in den Lymphdrüsen, den Lungen und Pleuren, sowie im Kleinhirn. Zunächst bietet dieser Fall (wie der oben erwähnte Lubarschs) große Ähnlichkeit mit dem meinen durch den sonst noch kaum beobachteten Sitz der Tumoren in der Lunge, anch die Multiplicität stimmt überein. Während aber in meinem Fall, wo die Tumoren im Kehlkopf und der Lunge saßen, ein eigentliches Metastasieren nicht angenommen zu werden braucht, handelte es sich im Falle Burks um augenscheinliche Metastasen, ein Verhalten, das bisher von Amyloidtumoren nicht bekannt war. 